\title{
The Mapping of Visual Space onto Foveal Striate Cortex in the Macaque Monkey ${ }^{1}$
}

\author{
B. M. DOW, ${ }^{2}$ R. G. VAUTIN, AND R. BAUER ${ }^{\star}$
}

Division of Neurobiology, Department of Physiology, School of Medicine, State University of New York, Buffalo, New York 14226 and

${ }^{\star}$ Abteilung fur Biologie IV der Universitat UIm, Oberer Eselsberg M 25/D, Postfach 4066, D-7900 UIm, West Germany

\begin{abstract}
Visuotopic maps of foveal striate cortex have been obtained by means of single cell recordings from four hemispheres in two awake, behaving macaque monkeys. The numbers of successful separate striate penetration sites in the four hemispheres were $42,58,81$, and 61 , for a total of 242. The resolution of the maps is $10 \mathrm{~min}$ of visual angle, nearly an order of magnitude finer than previous maps. No striate receptive field center was found more than 5 min into the ipsilateral visual field. The four maps were sufficiently compatible with one another that they could be combined into one. There are only minor magnification differences between the right and left hemispheres and between the upper and lower quadrants. There is a vertical/horizontal magnification anisotropy of about $1.5: 1$ in central foveal cortex (0 to $20 \mathrm{~min}$ ). The composite map can be approximated by the complex logarithmic equation, $w=7.7 *$ In $(x+i y+$ 0.33 ), where $w$ is expressed in millimeters and $x$ and $y$ are expressed in degrees.
\end{abstract}

Recent developments in the study of visual cortical organization in primates have indicated the need for a detailed description of the visuotopic organization of the primary visual cortex (striate cortex, V1). The details of a striate map are relevant to three independent areas of research. These are: (1) models of striate orientation column architecture (Braitenberg and Braitenberg, 1979; Dow and Bauer, 1984); (2) mathematical descriptions of the mapping of the retina onto striate cortex (Schwartz, 1980, 1983); and (3) examination of the principles governing the mapping of striate cortex onto extrastriate areas such as V2, V4, and MT (Zeki, 1969; Allman and Kaas, 1971, 1974; Van Essen, 1979; Weller and Kaas, 1983).

Although there are at present four published visuotopic maps of striate cortex in Old World monkeys (Talbot and Marshall, 1941; Daniel and Whitteridge, 1961; Guld and Bertulis, 1976; Tootell et al., 1982), none of the existing maps provides sufficient resolution in the foveal region. The map presented here has a resolution of $10 \mathrm{~min}$ of visual angle and is limited to the foveal region.

Some of our results have been published in a recent report describing visual magnification in the macaque monkey's foveal striate cortex (Dow et al., 1981). The present report, based on the

Received April 30, 1984; Revised September 14, 1984;

Accepted October 8, 1984

${ }^{1}$ This work was supported by National Institutes of Health Grants EY02349 and 5T32 EY07019. We thank James Stamos, Julie Lakatos, and Margaret M. Edlich for help with illustrations, and Linda Smith for typing.

${ }^{2}$ To whom correspondence should be addressed. same data, provides additional details concerning the actual visuotopic map and its relationship to histological landmarks.

\section{Materials and Methods}

Data collection procedures have been described in detail in our previous reports (Bauer et al., 1980, 1983; Dow et al., 1981). In brief, rhesus monkeys were trained to fixate a light-emitting diode (LED) attached to the back of a translucent tangent screen. Receptive field mapping was done during the 1 to 6-sec fixation periods imposed by the behavioral task. A joystick was used to control the position of a variable-sized rectangle of light projected onto the tangent screen. Some receptive fields were also mapped quantitatively with the stimulus position under computer control (see Fig. 3 in Dow et al., 1981; B. M. Dow and R. G. Vautin, unpublished data).

Recordings were obtained with glass-coated platinum-iridium electrodes positioned within a recording cylinder $(2 \mathrm{~cm}$ in diameter) mounted chronically on the skull over the unopened dura. Microelectrodes were positioned by means of a double-eccentric coordinate system designed by Evarts (1966) and were advanced under hydraulic control (Narishige MO-9). Data used in this report were taken trom penetrations in four cylinders (left and right hemispheres of two monkeys). The positions of penetration sites in recordingcylinder coordinates on a 1-mm grid are shown in Figure 1.

A total of 269 different penetration sites were successfully assigned visual receptive field locations in the present study (some sites included several penetrations made at different times). The numbers of successful penetration sites in the four cylinders were $43,63,82$, and 81 . Penetration sites containing identified electrolytic lesions ( $10 \mu \mathrm{A}$ of electrode-positive current for $1 \mathrm{~min}$ ) are indicated by small $x$ 's in Figure 1, with unlesioned penetration sites indicated by solid circles.

The numbers of penetrations marked with lesions in the four cylinders were 4, 5, 6, and 4 . In addition, following sacrifice, perfusion, and removal of the dura, three sharpened, stainless steel capillary tubes (pins) were filled with India ink and seated in each cortical hemisphere at known cylinder locations using the microdrive. Photographs of the brain surface were taken with the camera aligned so that the India ink pins appeared as dots on the cortical surface. In Figure 1, the solid circles with vertical lines through them indicate the locations of India ink pins in the four cylinders. The pins were left in the brain during skull dissection, as previously described (Bauer et al., 1980, 1983), and used to establish a plane of section for histology. The four cylinders in Figure 1 have been positioned such that, for each cylinder, the plane of section corresponds to horizontal on the page.

Each brain was then blocked, sectioned at $32 \mu \mathrm{m}$, and stained for Nissl substance (cresyl violet) and fibers (Loyez). Details concerning histological reconstruction will be described under "Results."

\section{Results}

General procedure. The recording sites in Figure 1 are all located with respect to the $x, y$ coordinates within each recording cylinder. In a previous report (Dow et al., 1981) these cylinder coordinates and associated receptive field data were used to determine striate cortical magnification as a function of visual field eccentricity. An essential task of the present study was to locate the recording sites with respect to brain landmarks, thus transferring retinotopic maps from the recording cylinders to the brain surface. Because of the large numbers of penetrations in these monkeys it was not possible 

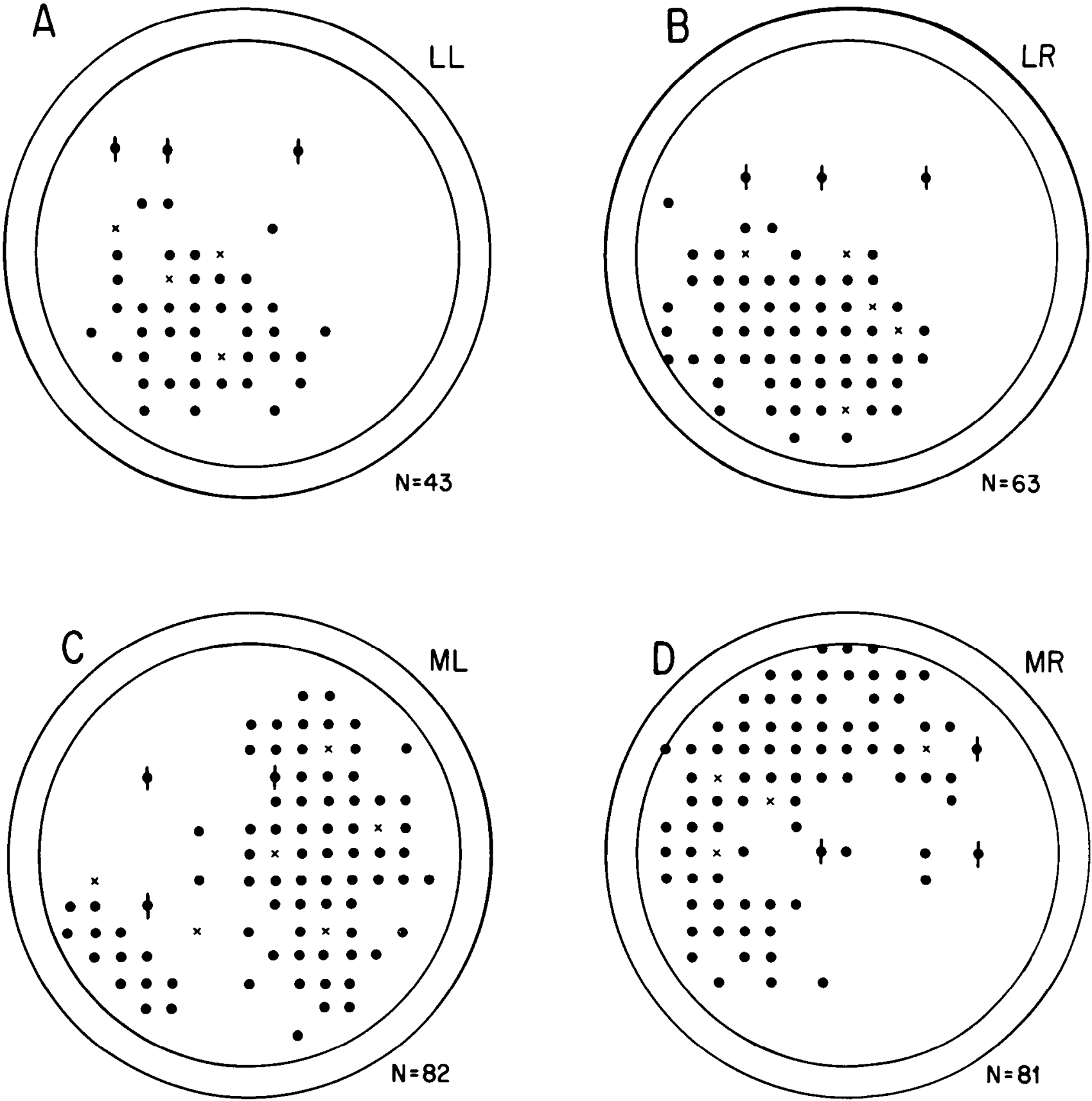

Figure 1. Microelectrode placement sites within the recording cylinders for the four hemispheres of the present study. For $A, B, C$, and $D$ the outer circle marks the cylinder wall; the inner circle indicates the region accessible to microelectrodes. Grid points are separated by $1 \mathrm{~mm}$. Solid circles with vertical bars through them represent the sites at which sharpened stainless steel capillary tubes were placed in the brain following perfusion of the animal and removal of the dura. Regular solid circles are sites of successful microelectrode penetrations, with $x$ 's indicating marked penetrations (see the text). Letters at the upper right identify hemispheres; numbers at the lower right indicate the number of penetration locations in each cylinder.

to reconstruct each penetration individually within the tissue. Instead, an alternative method was used which takes advantage of the large numbers of penetrations.

The procedure involved reconstructing each tissue block from its component sections, making use of surface landmarks to reconstitute an outline drawing of the local brain surface. On each reconstructed block the exact location of the recording cylinder was specified by the positions of electrolytically marked microelectrode tracks and India ink pin tracks. The drawing of the reconstructed block was then superimposed on a photograph of the brain surface (for example, see Fig. 9A) taken at the time of perfusion. The India ink pin tracks and major surface sulci present in the reconstructed block drawings and also visible in the brain photographs were used to achieve proper alignment.

Some of the steps in construction of a visuotopic map from each cylinder map have already been described (Dow et al., 1981). Further details are provided below. The critical step in the present procedure is the use of the cylinder coordinate system as a link between the tangent screen and the cortical surface. Limitations of the method and possible sources of error will be described under "Discussion."

Block reconstructions. Precise blocking of the brain is essential for three-dimensional tissue reconstruction. In the present study this was achieved by first establishing a primary section plane in the stereotaxic apparatus (see above), and then establishing additional (secondary and tertiary) stereotaxic planes perpendicular to the 

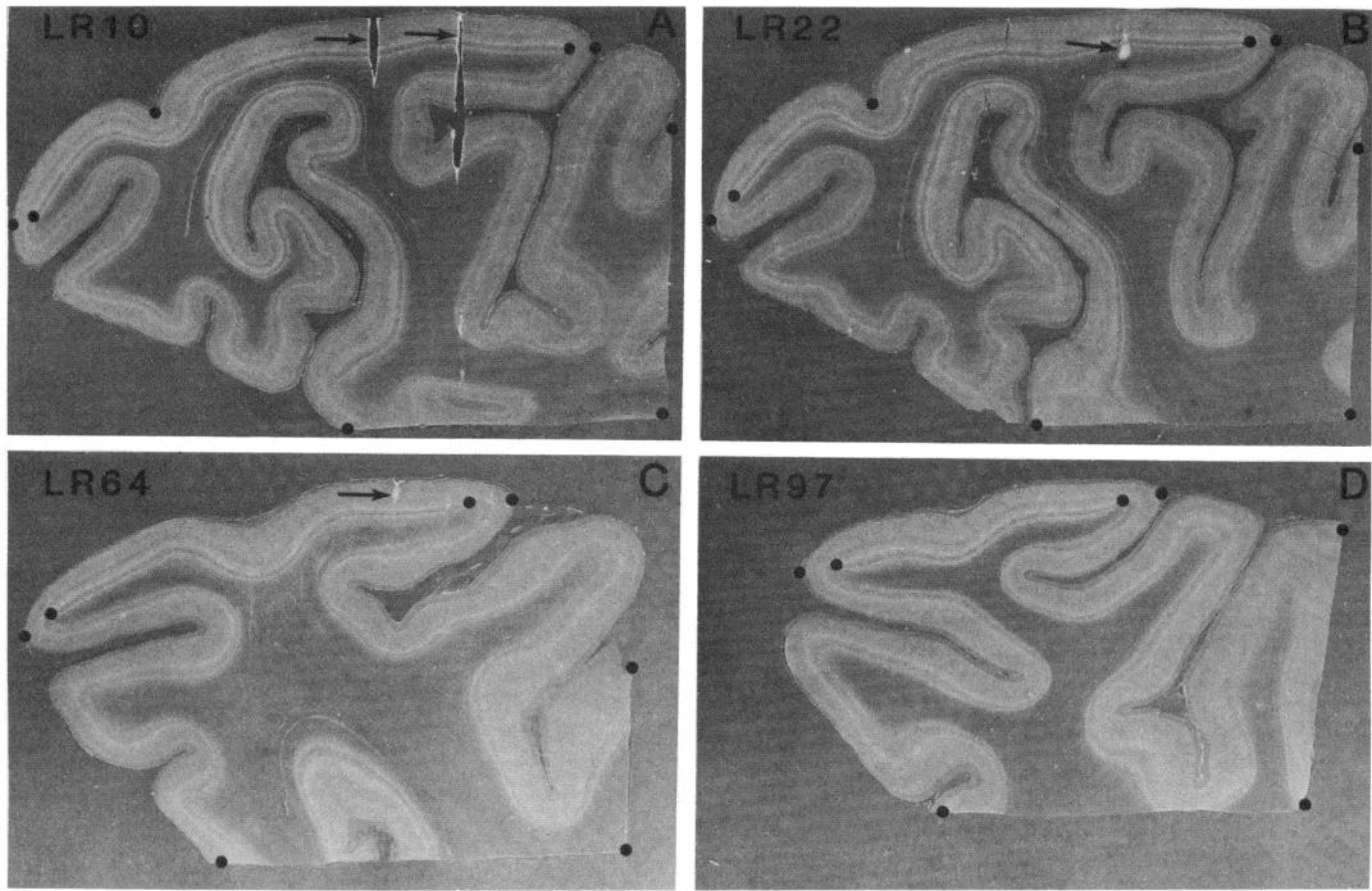

Figure 2. Representative serial sections $(A, B, C, D)$ from a single hemisphere (LR). Each section was $32 \mu \mathrm{m}$ thick. Arrows indicate stainless steel pin tracks $(A)$ and microelectrode lesion sites $(B$ and $C)$. Black dots are explained in the text.

section plane. The secondary and tertiary planes are best established with the primary section plane of the tissue block sitting on a flat surface. These additional planes provide a right-angle corner on each section which can then be used for alignment. Several sections obtained from one hemisphere in the present study are illustrated in Figure 2. The pictures are negative images printed directly from slides mounted in an enlarger. Note the right-angle corners at the lower right of each section. The arrows in Figure $2 A$ point to tracks left by the India ink pins. The presence of two pin tracks in the same section ( $L R$ 10) provides evidence that the primary section plane was reasonably parallel to the plane established by the pins. Furthermore, the two sides of the right-angle corner in Figure $2 A$ are approximately parallel and perpendicular, respectively, to the pin tracks. In Figure 2, $B$ and $C$, the arrows point to electrolytic lesions made along microelectrode recording tracks. Microelectrode tracks were in general parallel to one another and to India ink pin tracks, as is illustrated here. Occasional aberrant penetrations were seen, however. Some of these may have resulted from irregularities in electrode tip shape; others may have resulted from electrode deflection by the dura or pial blood vessels.

Once all marked penetrations had been located in the tissue, a series of photographs such as those of Figure 2 was prepared for each tissue block, care being maintained to preserve constant magnification for an entire series. In two hemispheres (LL and LR) photographs were prepared from sections located $384 \mu \mathrm{m}$ apart (every 12th 32- $\mu \mathrm{m}$ section). In the other two hemispheres (ML and MR) photographs were prepared from sections located $896 \mu \mathrm{m}$ apart (every 28th 32- $\mu \mathrm{m}$ section). Each set of photographs was then marked with black dots (see Fig. 2) to highlight the right-angle corner (three dots), the two V1-V2 borders (two dots), and the locations of major tissue curvature or sulci (three dots in Fig. 2, $A$ and $B$, two dots in Fig. 2, $C$ and $D$ ), as seen from above.

Using the right-angle corner dots for alignment, the remaining black dots from each photograph were transferred to millimeter graph paper. To reconstruct a given tissue block, one line on the graph paper was assigned to each photograph. Line assignments for successive photographs were established by the photographic enlargement ( $\times 8 \pm 5.6 \%$ in this study). The procedure is similar to one used by Hubel and Wiesel (1972) to reconstruct terminal degeneration in striate cortex following lesions in the lateral geniculate nucleus.

The results of such a reconstruction procedure conducted on the four hemispheres of the present study are illustrated in Figure 3. Perhaps the process can be best understood by looking first at Figure $3 B$ and locating the four sections of Figure 2 on the vertical scale (sections $10,22,64$, and 97 ). It should be noted here that the section numbers in this figure and in Figure 2 refer to a series of Nissl-stained sections at $128-\mu \mathrm{m}$ intervals. The solid circles in Figure 2 are shown as open circles in Figure 3. India ink tracks and electrolytic lesions are indicated by the same symbols as those used in Figure 1.

Although there is inevitably some distortion of surface features when they are projected in this way (for example, the brain surface falls away more sharply to the left in Figure $2 B$ than in Figure $2 D$ ), the reconstructed "pictures" in fact resemble rather closely actual photographs taken of the respective hemispheres prior to blocking (see below). A particular advantage of such projections is that they enable one to see buried landmarks (the V1/V2 border, for example) which are not visible on a surface photograph. The method is especially useful for establishing surface correlates of buried extrastriate regions (unpublished data). 

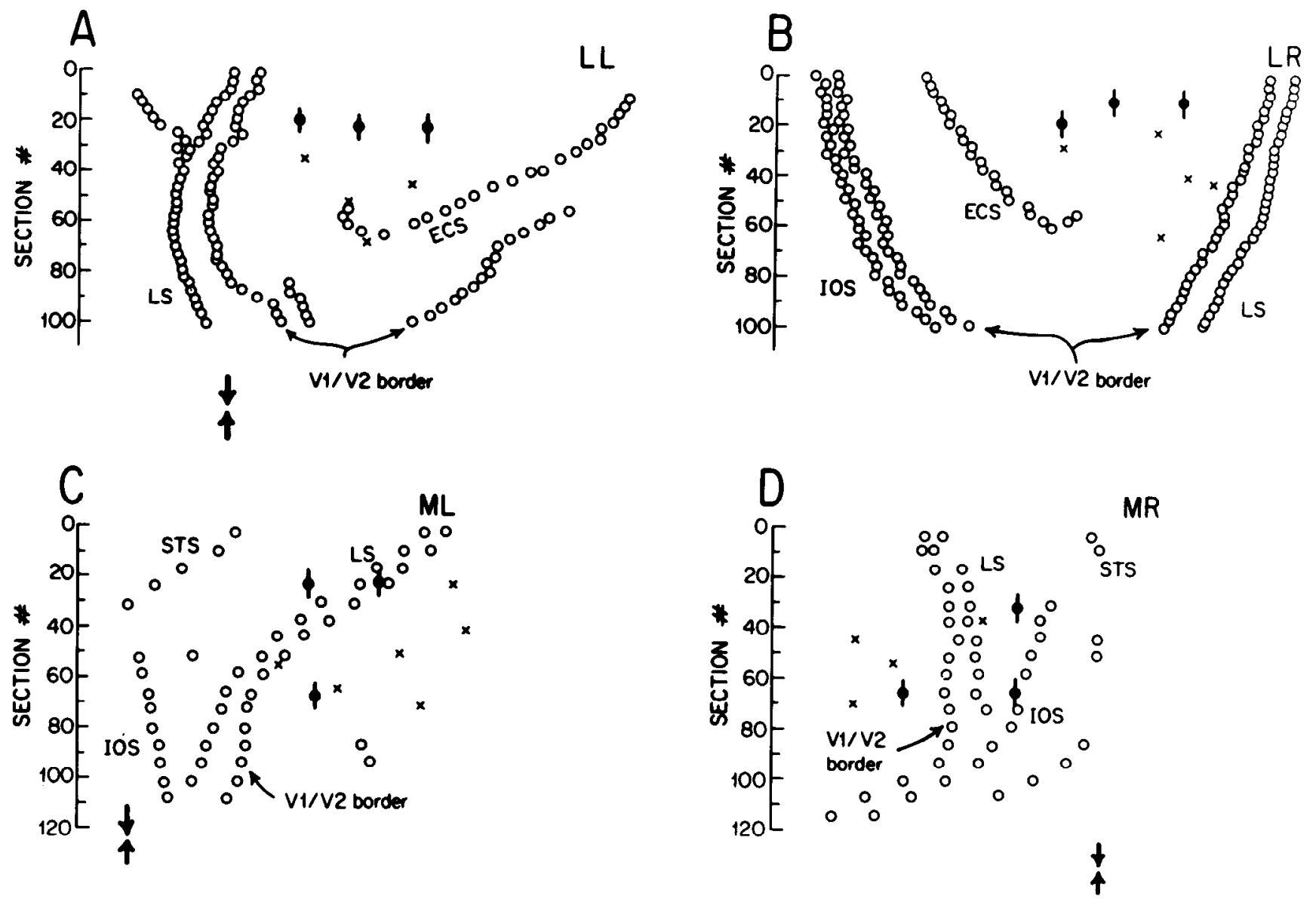

Figure 3. Reconstructions of the four tissue blocks in the present study showing tissue landmarks in relation to marked tissue. Every 12 th $(A$ and $B$ ) or 28 th ( $C$ and $D)$ 32- $\mu \mathrm{m}$ section was plotted on 1-mm graph paper (section numbers refer to a series of Nissi-stained sections at 128- $\mu \mathrm{m}$ intervals). Actual stainless steel pin tracks and microelectrode lesion sites are indicated by the same symbols that were used in Figure 1 to indicale inlended sites. Pairs of facing arrows below each reconstruction indicate the location on the horizontal axis of the right-angle corner used to align the tissue sections (see the text). In $A$, each section was rotated $25^{\circ}$ counterclockwise about this corner in an attempt to reproduce the cylinder angle with respect to the brain surface, thus making penetration tracks appear nearly vertical on the graph paper used for reconstruction. ECS, external calcarine sulcus; IOS, inferior occipital sulcus; $L S$, lunate sulcus; STS, superior temporal sulcus.

In one hemisphere of the present study (LL) the recording cylinder was mounted onto the skull at a $25^{\circ}$ angle from vertical. The reconstruction of this hemisphere was noticeably skewed comparcd with the other three hemispheres. Thus, for example, the projection of the external calcarine sulcus (ECS) is closer to the V1/N2 border in Figure $3 A$ than in Figure $3 B$. Two marked penetrations in hemisphere $L L$ had angles of $27.6^{\circ}$ and $42^{\circ}$ to the surface normal. In hemispheres $\mathrm{LR}, \mathrm{ML}$, and MR (Fig. 3, $B$ to $D$ ), the cylinder angle to the skull was 0 to $5^{\circ}$, and penetration angles to the surface normal averaged $9.4^{\circ}, 17.8^{\circ}$, and $14.4^{\circ}$, respectively (see Bauer et al., 1983, Table 2). It is clearly desirable in studies such as these to have the recording cylinder mounted as nearly orthogonal as possible to the tissue surface being reconstructed.

It is useful to compare Figure 3 with Figure 1. Using the India ink pins which appear in both figures as a guide, the cylinders (Fig. 1) and reconstructed blocks (Fig. 3) have been aligned to facilitate comparisons. However, the magnifications of Figures 1 and 3 are not matched. Since the India ink pins were inserted after perfusion (and removal of the dura), their relationship to other cylinder locations has probably been distorted somewhat by tissue shrinkage and settling of the brain. More significant for a detailed comparison between Figures 1 and 3 are the locations of the electrolytic lesions $(x$ 's). Figure 1 shows the $x$ 's in cylinder coordinates, and Figure 3 shows where the lesions actually occurred in relation to one another as well as to natural tissue landmarks. All $x$ 's except one (in MR) were located within the confines of $\mathrm{V} 1$. The extrastriate lesion in MR was placed in V4 at a site where many color cells were recorded (Dow et al., 1979). One lesioned electrode track in ML sits just on the V1/N2 border. Photographs of this track (ML 85) and of two others (ML 79, LR 135) have already been published (Dow et al., 1981; Bauer et al., 1983). Among electrolytic lesions ( $x$ 's) within a given cylinder, the general spatial pattern is recognizable, although the arrays in Figures 1 and 3 are in no case superimposable, even when the magnifications have been matched. In general, electrolytic lesions were within $\pm 1 \mathrm{~mm}$ of where they were supposed to be (see "Discussion").

Visuotopic maps. Successful striate recording sites and receptive field center locations at each recording site are illustrated in Figures 4 and 5 for the two monkeys of the present study. Extrastriate penetrations were excluded on histological grounds (see below). Striate recording sites, of which there were $42,58,81$, and 61 , respectively, are shown in Figure 4, $A$ and $C$, and Figure 5, $A$ and $C$. Receptive field center locations at these recording sites are shown in Figure 4, $B$ and $D$, and Figure $5, B$ and $D$, respectively, each point representing the center of the aggregate receptive field (arithmetic mean of the individual receptive field centers) for a single striate penetration. These data points were used previously to obtain an inverse magnification curve for foveal striate cortex (Dow et al., 1981).

When more than one successful penetration was made at the same recording site, separate points were plotted for each penetration. These are shown as open circles in Figures 4 and 5. The 
A

Column

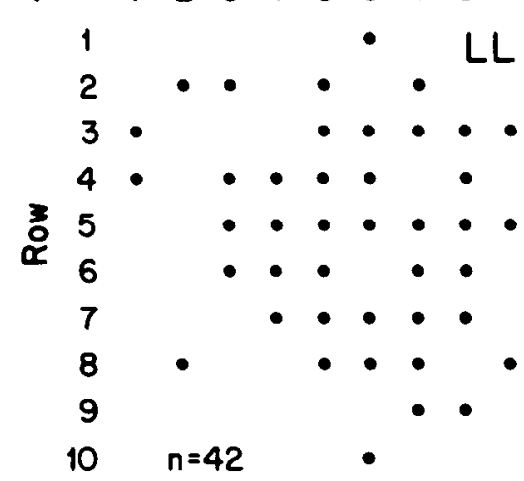

Minutes
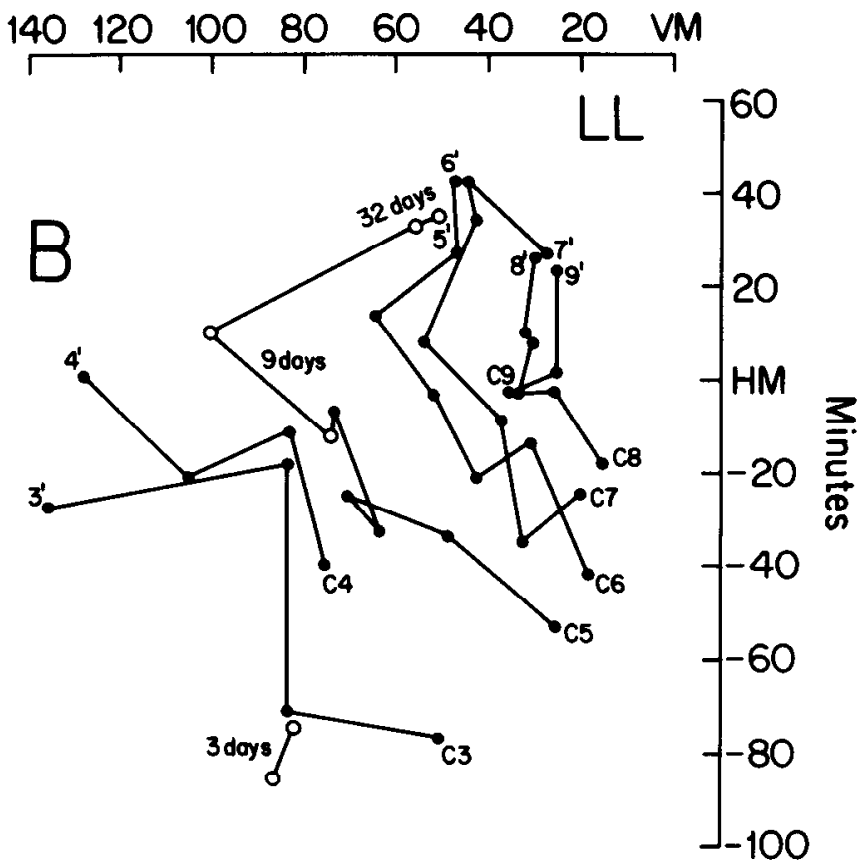

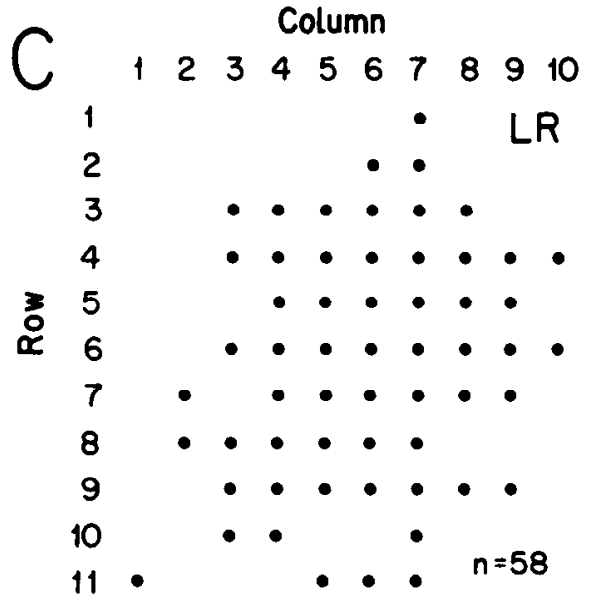

Minutes
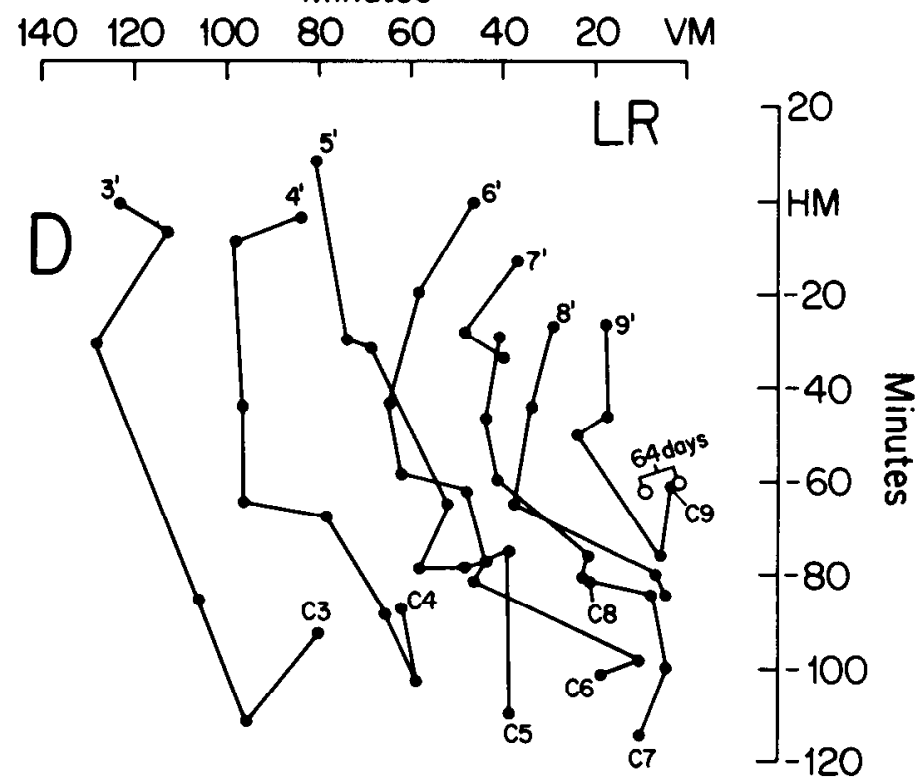

Figure 4. Recording sites and aggregate receptive field centers for hemispheres $L L(A$ and $B$ ) and $L R(C$ and $D)$. In $A$ and $C$ recording sites are designated in cylinder coordinates as columns and rows. All columns including at least three points are represented as connected points in $B$ and $D$. $H M$, horizontal meridian; VM, vertical meridian. Open circles indicate pairs of penetrations at the same recording site, separated in time (days) by the indicated interval.

number just adjacent to each pair of open circles refers to the interval in days between the two penetrations at this recording site. The separation between open circles, which can be directly measured in minutes of visual angle, and computed in millimeters of cortical tissue on the basis of the magnification factor relationship (see "Discussion"), gives an indication of the reproducibility of our electrode positioning over extended periods of time in these chronic experiments. In many instances the pairs of points were close enough together to be within the range of receptive field scatter seen in a single penetration (see "Discussion"). There is no correlation between time interval and separation distance, indicating that the variability is probably due to differences in electrode shape and variations in electrode tip deflection on passing through the dura, rather than progressive changes in the position of the brain within the skull.

For the sake of convenience, all receptive field center locations obtained from left hemisphere recording sites have been mirrorimage reversed about the vertical meridian. Cylinder grids have been adjusted so as to conform as closely as possible to one another.
Here it may be useful to compare Figures 4, $A$ and $C$, and $5, A$ and $C$ to Figure 1. Hemisphere MR (Figs. $1 D$ and $5 C$ ) has retained the same orientation in both figures. Hemisphere ML (Figs. $1 C$ and $5 A$ ) has been mirror-image reversed. Hemisphere LR (Figs. $1 B$ and $4 C$ ) has been rotated $90^{\circ}$ counterclockwise, and hemisphere LL (Figs. $1 A$ and $4 A$ ) has been mirror-image reversed and then rotated $90^{\circ}$ counterclockwise.

Each column containing at least three grid points in Figures $4, A$ and $C_{1}$ and $5, A$ and $C$ is shown in Figure $4 B, 4 D, 5 B$, or $5 D$ as a series of connected points, the starting point (beginning at the top in Fig. $4 A, 4 C, 5 A$, or $5 C$ ) indicated as $C n$, the ending point as $n^{\prime}$, where $n$ is the column number. The progression of the lines across visual space is generally quite regular, although there are irregularities in the locations of individual points along each line. In the centralmost portions of the ML and MR maps, the individual lines become difficult to resolve, not only because of the increased magnification at these eccentricities, but also because receptive field size and scatter decrease at slower rates than inverse magnification, as previously reported (Dow et al., 1981) and more recently confirmed (Van Essen 
A

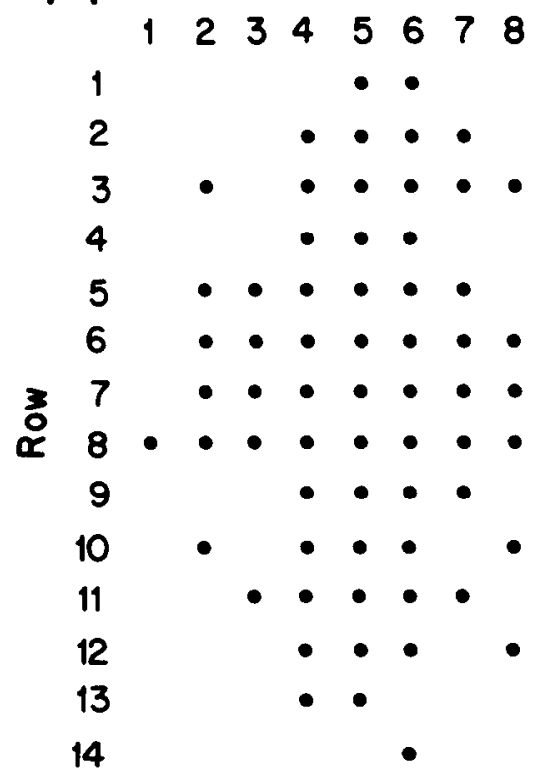

Column
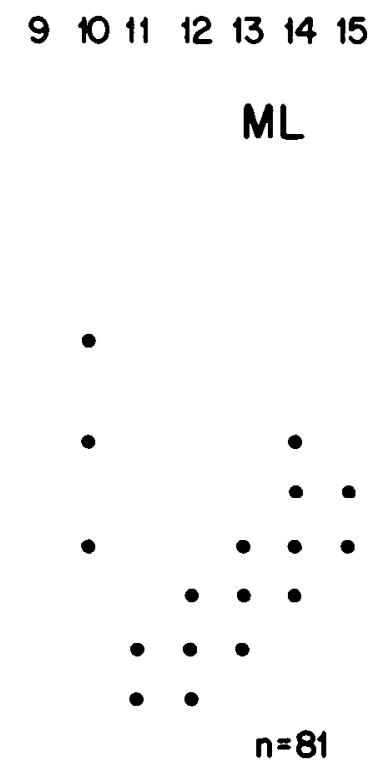

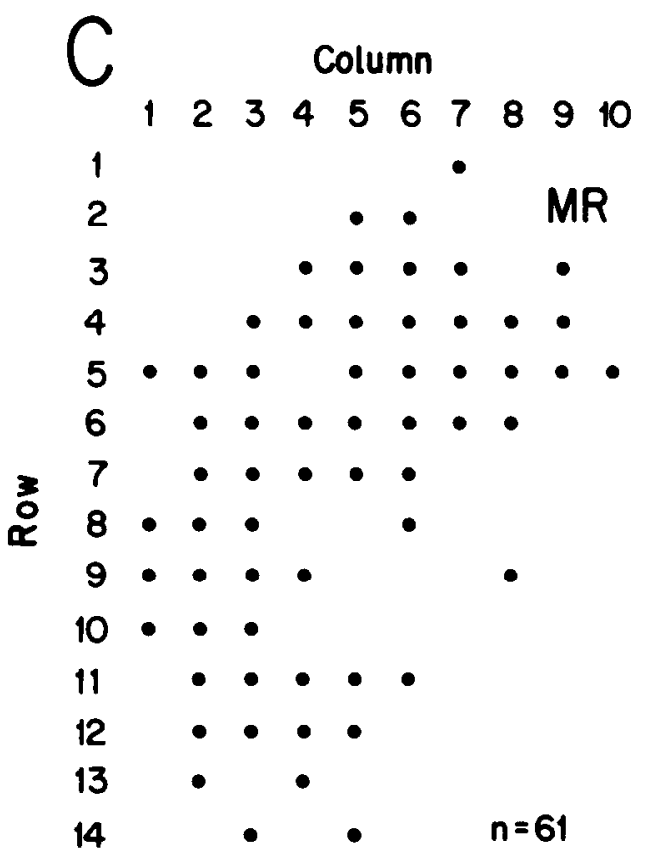

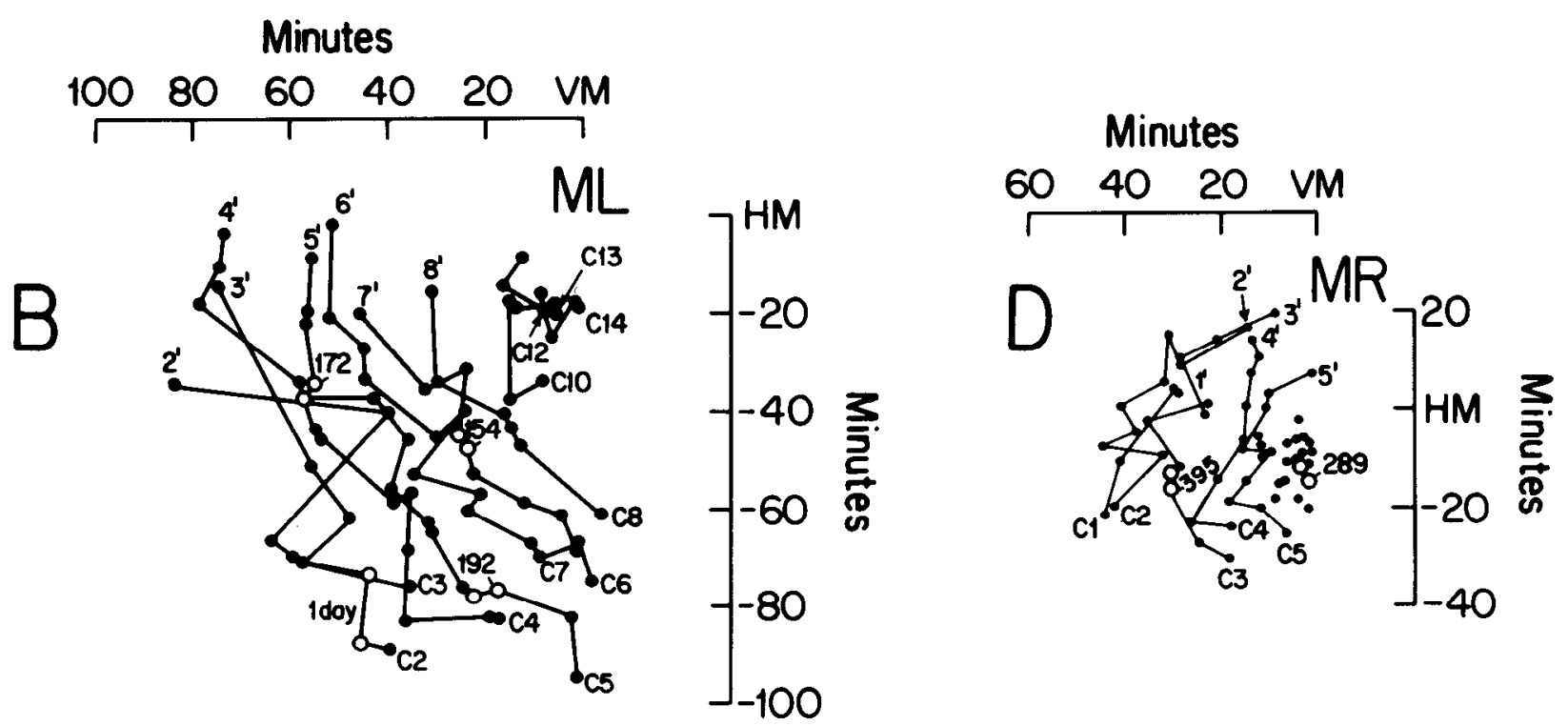

Figure 5. Recording sites and aggregate receptive field centers for hemispheres ML ( $A$ and $B$ ) and MR ( $C$ and $D$ ), using the same format as in Figure 4. except that columns 10 to 14 in $A$ are shown in $B$ without terminators $\left(n^{\prime}\right)$, and columns 6 to 9 in $C$ are shown in $D$ without connecting lines in order to preserve visibility.

et al., 1984). Nevertheless, orderliness of the map seems to be present even at the lowest eccentricities.

It is particularly noteworthy that few aggregate field centers are located in the ipsilateral visual field, and none are located more than $5 \mathrm{~min}$ into the ipsilateral field. Given receptive field sizes and aggregate field sizes in this part of striate cortex (Dow et al., 1981), the total encroachment of foveal striate fields into the ipsilateral visual field is not more than about 20 to 25 min of arc. Note also that most of the data points are in the inferior field. This is largely due to the fact that cylinders were placed so as to provide access to extrastriate regions located in the lunate sulcus.

The next step in the analysis of the data was to place the receptive field data points for each hemisphere into 10-min bins, centered on azimuth lines $0,10 \mathrm{~min}, 20 \mathrm{~min}, 30 \mathrm{~min}$, etc., and on elevation lines $+20 \mathrm{~min},+10 \mathrm{~min}, 0,-10 \mathrm{~min},-20 \mathrm{~min},-30 \mathrm{~min}$, etc. Each recording site in a given cylinder was then assigned both an azimuth and an elevation bin number. Figure 6 shows balloon diagrams obtained by circling all except the most aberrant recording sites in the same bin, with azimuth bins shown as thick lines and elevation bins shown as thin lines.

Using the balloon diagrams as a guide, the azimuth and elevation lines for each 10-min bin were then drawn onto each cylinder grid. The resulting maps for the four cylinders are shown in Figure 7. All recording sites in Figure 1 are included in Figure 7, with striate sites represented as solid circles, and extrastriate sites as either open circles or asterisks. Open circles in Figure 7 indicate penetrations in which we failed to isolate striate cells before traversing white matter and entering buried extrastriate cortex. Asterisks indicate penetrations restricted to extrastriate cortex. Some of these penetrations entered cortex within V2; others entered cortex within V4. Extrastriate penetrations will not be further discussed here.

Figure 7 permits useful comparisons to be made between hemi- 
A

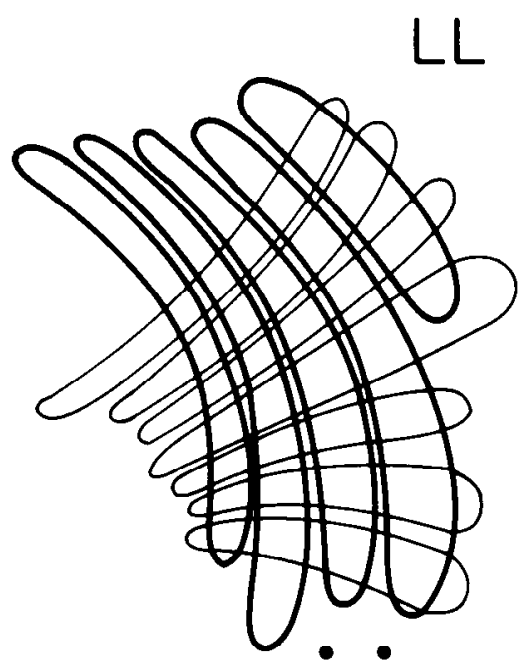

$1 \mathrm{~mm}$
B

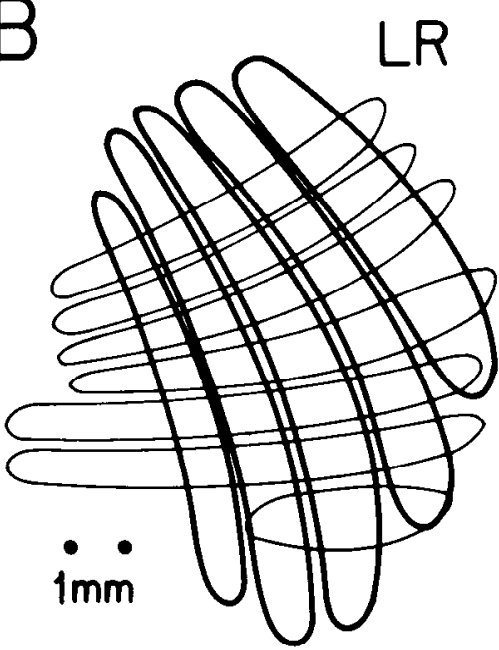

D
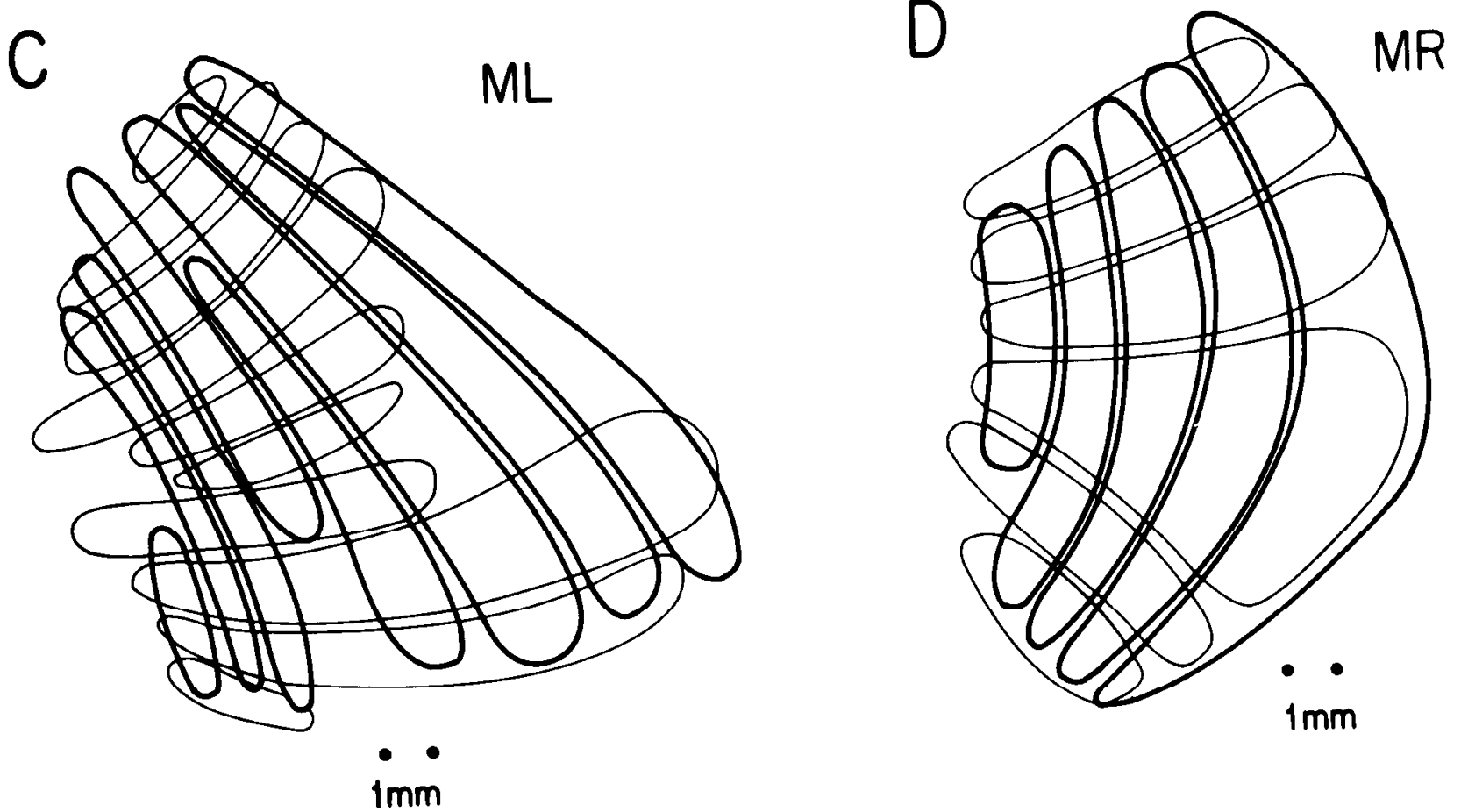

Figure 6. Balloon maps of the recording cylinders for the four hemispheres $(A$ to $D)$ of the present study. Each balloon represents a 10-min isoazimuth or iso-elevation bin, the former indicated by heavy lines, the latter by light lines. All maps are shown at the same scale.

spheres and between monkeys. One can compare magnifications by finding squares that are common to more than one map. For example, the eight squares bounded by elevation lines -10 and -30 and azimuth lines $0(\mathrm{VM})$ and 40 (the lines are thickened for greater visibility) are common to $\mathrm{ML}$ and MR. The sizes of comparable squares in the two hemispheres are generally similar to one another, given some uncertainty as to the exact placement of the lines. Linear magnification along the -20 -min iso-elevation line that runs down the middle of this eight-square zone measures 11.25 $\mathrm{mm} /$ degree in MR and $11.40 \mathrm{~mm} /$ degree in ML, a ratio of 0.98 .

$\mathrm{LL}$ and $\mathrm{LR}$ can be compared between elevation lines -20 and $-30 \mathrm{~min}$ and azimuth lines 20 to $50 \mathrm{~min}$. Here the areas are somewhat smaller for $L L$ than for LR, perhaps to be expected from the $25^{\circ}$ tilt of the $\mathrm{LL}$ cylinder (see above). Linear magnification along the -20 -min iso-elevation line in this region measures $9.0 \mathrm{~mm} /$ degree in $L L$ and $10.8 \mathrm{~mm} /$ degree in $L R$, a ratio of 0.83 .
The two squares bounded by elevation lines -20 and $-30 \mathrm{~min}$ and azimuth lines 20 to $40 \mathrm{~min}$ are present in all four cylinders (shaded regions in Fig. 7). These squares are largest in ML, smallest in LL, and intermediate in LR and MR. Linear magnification on the -20 -min iso-elevation line between azimuths 20 and $40 \mathrm{~min}$ is 6.6 $\mathrm{mm} /$ degree for $L L, 7.8 \mathrm{~mm} /$ degree for $L R, 8.1 \mathrm{~mm} /$ degree for $M R$, and $9.6 \mathrm{~mm} /$ degree for $M L$. Here the ratio between $L L$ and $L R$ is 0.85 , and between MR and ML it is 0.84 . With one monkey showing greater magnification in the right hemisphere and the other monkey greater magnification in the left hemisphere, there is some indication here that neither the right nor the left foveal striate region is consistently larger than its opposite counterpart, although the comparison is based on only two monkeys.

Because of the magnification difference between foveal striate regions of the two hemispheres in the same monkey, it would seem most relevant to compare either larger or smaller sides when com- 
A

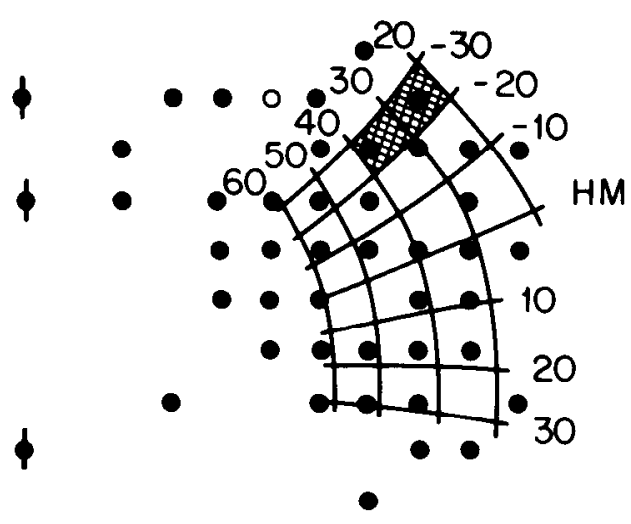

C

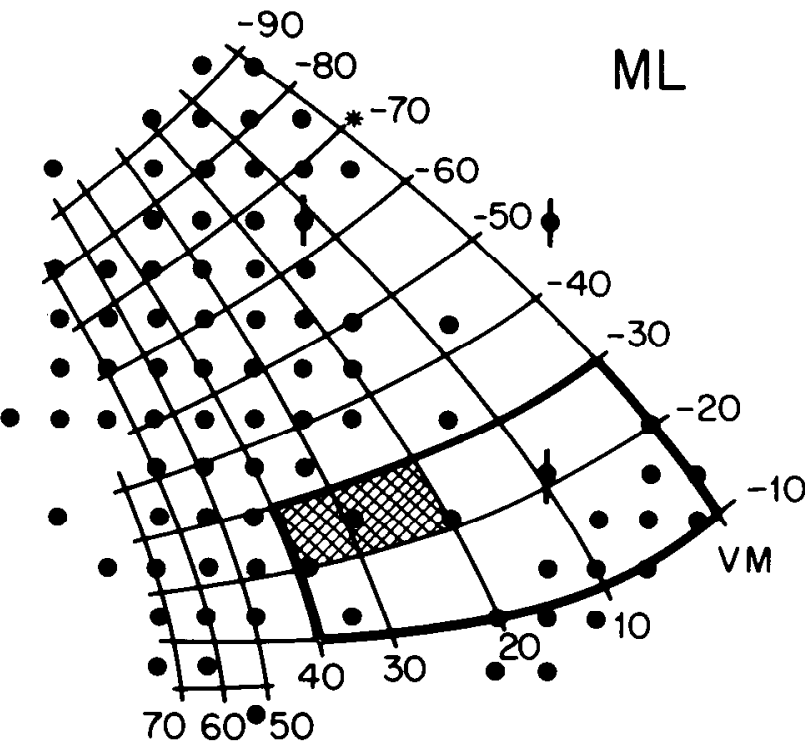

Q

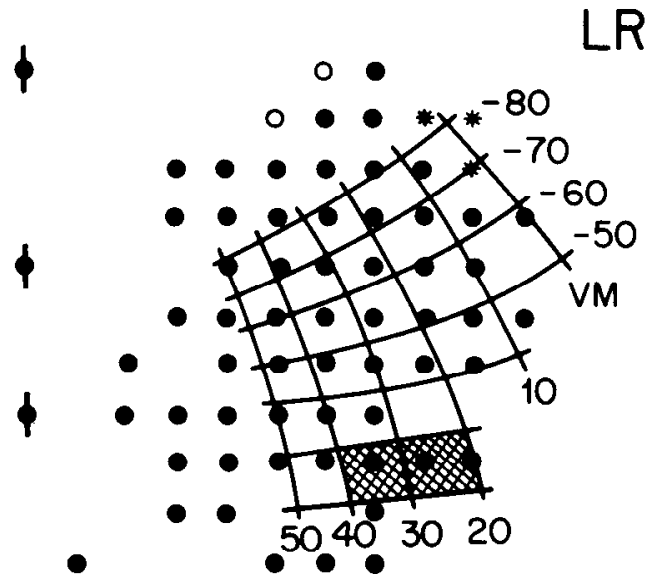

D

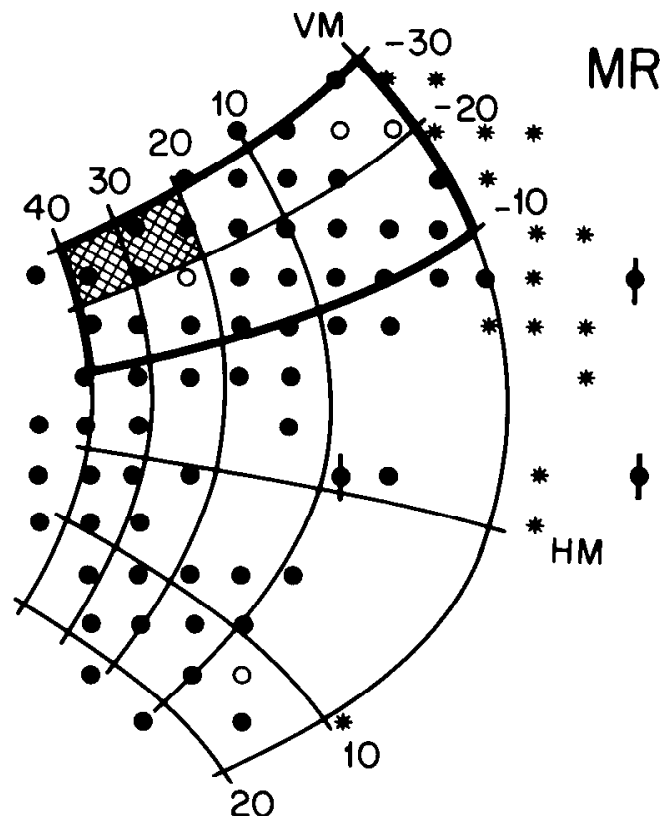

Figure 7. Visuotopic maps of the recording cylinders for the four hemispheres of the present study. Azimuth and elevation lines for each cylinder map are the centers of 10 -min bins (e.g., 5 to $15 \mathrm{~min}, 15$ to $25 \mathrm{~min}$. . .) for the respective data points of Figures 4 and 5 . All points in the cylinder maps of Figure 1 are reproduced here, with rotations and striate/extrastriate designations as described in the text, in relation to Figures 4 and 5 . Shaded regions in $A$ to $D$ have the same visual field coordinates. Heavy lines in $C$ and $D$ enclose regions with the same visual field coordinates.

paring one monkey with another. For the two monkeys of the present study the ratio of linear magnifications (in the measured region) was 0.81 for larger sides and likewise 0.81 for smaller sides. This is about the same as the ratio between sides in a given monkey. The ratio between the largest and smallest linear magnifications ( $L L$ versus $\mathrm{ML}$ ) at this site in the present study was 0.69 .

It would be of interest to know whether the total V1 surface area differed for the four hemispheres and two monkeys of the present study. Anatomical studies have typically reported a range of $V_{1}$ surface area values for macaques of 900 to $1300 \mathrm{~mm}^{2}$, possibly reflecting age, sex and species differences (see Weller and Kaas, 1983, for a recent review). Van Essen et al. (1984) recently reported a mean of $1195 \mathrm{~mm}^{2}$, based on 31 hemispheres from 24 Macaca fascicularis monkeys weighing 1.5 to $3.0 \mathrm{~kg}$. Both monkeys in the present study were young rhesus males. At the time of sacrifice one monkey (LL, LR) weighed $5.6 \mathrm{~kg}$, while the other (ML, MR) weighed $6.3 \mathrm{~kg}$. At the time of sacrifice the heavier monkey was also about 1 year older than the other monkey.

Cylinder to brain. The transfer of a visuotopic map from cylinder coordinates (Fig. 7) to the brain surface requires a brain photograph for each hemisphere obtained with a camera angle comparable to that of the recording cylinder. This was accomplished by photographing the brain prior to blocking, with the India ink pins viewed straight on, so that they appeared as black dots on the brain surface.

The actual transfer from cylinder map to brain surface was accomplished with the help of the block reconstructions (Fig. 3) as templates. The block reconstructions contain information about the location of cylinder landmarks (electrolytic lesion sites, India ink pin tracks) as well as brain surface landmarks (sulci, pins). Each block reconstruction (Fig. 3) was photographed, and the negative was projected from an enlarger onto the appropriate cylinder map (Fig. 7). The enlarger was adjusted until an approximate size match had been achieved. The amount of magnification required for this size match in fact provides a very convenient and accurate measure of tissue shrinkage ( $15 \%$ in the present study).

A print was then made of the enlarged block reconstruction, and a negative of the brain photograph was projected from the enlarger onto the block reconstruction print. Next, a print was made of the brain photograph at the proper enlargement. Finally, with all prints the same size, it was then a simple matter to transfer the visuotopic 


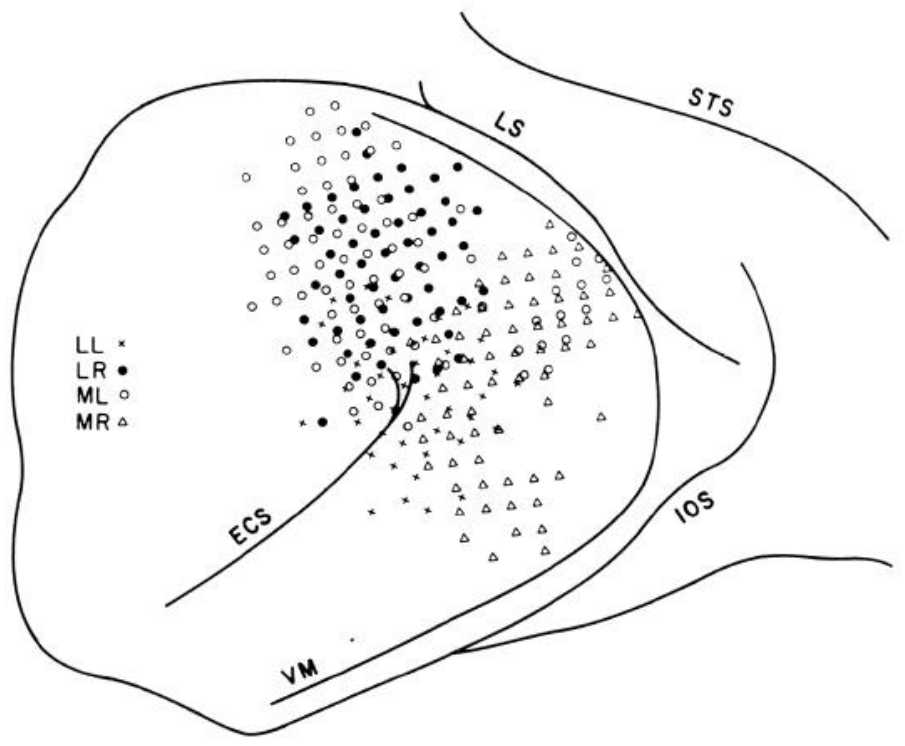

Figure 8. Recording sites for the four hemispheres of the present study superimposed upon the tracing of a surface photograph from a single hemisphere. Different symbols are used for each hemisphere: LL, LR, ML, and MR. ECS, external calcarine sulcus; IOS, inferior occipital sulcus; LS, lunate sulcus; STS, superior temporal sulcus; VM, vertical meridian.

map from cylinder coordinates to the brain surface with a light box, using the block reconstruction for alignment.

In the present study, despite some differences in magnification in the four hemispheres, it seemed reasonable to attempt a synthesis of the four maps into one, recognizing that interested readers would in any case have the individual maps of Figure 7 to refer to. The striate recording sites of Figure 7 were therefore all superimposed upon the tracing of a surface photograph from a single hemisphere, as illustrated in Figure 8 (LR was used as the representative hemisphere). In order to accomplish this synthesis, the brain reconstructions in Figure 3 and the cylinder maps in Figure 7 were all viewed together on a light box and made to conform as closely as possible to one another. The precise specification of the relative locations of the four cylinder maps on a single hemisphere permitted the construction of a single composite map of all recording sites (Fig. 8) as well as a single composite visuotopic map (Fig. 9A). An artist's tracing of the hemisphere is used in Figure $9 A$ to provide greater detail than is available in either an outline tracing or an actual photograph. Because of uncertainties concerning the tissue in the vicinity of the ECS it was decided not to extend the map through this region. It was also decided not to extend the map beyond 60 min of negative elevation, since, in relation to the camera's perspective, the tissue surface falls away from a flat plane beyond $60 \mathrm{~min}$. A three-dimensional model would be necessary to illustrate the details of the map at greater eccentricities.

To appreciate the precise distortions that are present in the foveal striate map shown in Figure $9 A$, it is useful to compare the map with Figure $9 B$, which is the corresponding region of visual space. As previously described (Dow et al., 1981), the inverse of the size (square root of the area) of each distorted square in Figure $9 \mathrm{~A}$ plotted against its center eccentricity gives a series of data points for an inverse magnification function. Of particular relevance in the present context is the issue of magnification anisotropy in the upper versus the lower quadrants and along the horizontal and vertical meridia. There is in fact little if any upper versus lower quadrant anisotrophy in Figure 9A, although admittedly fewer data points were obtained from the upper quadrant (see Figs. 4 and 5). There is, on

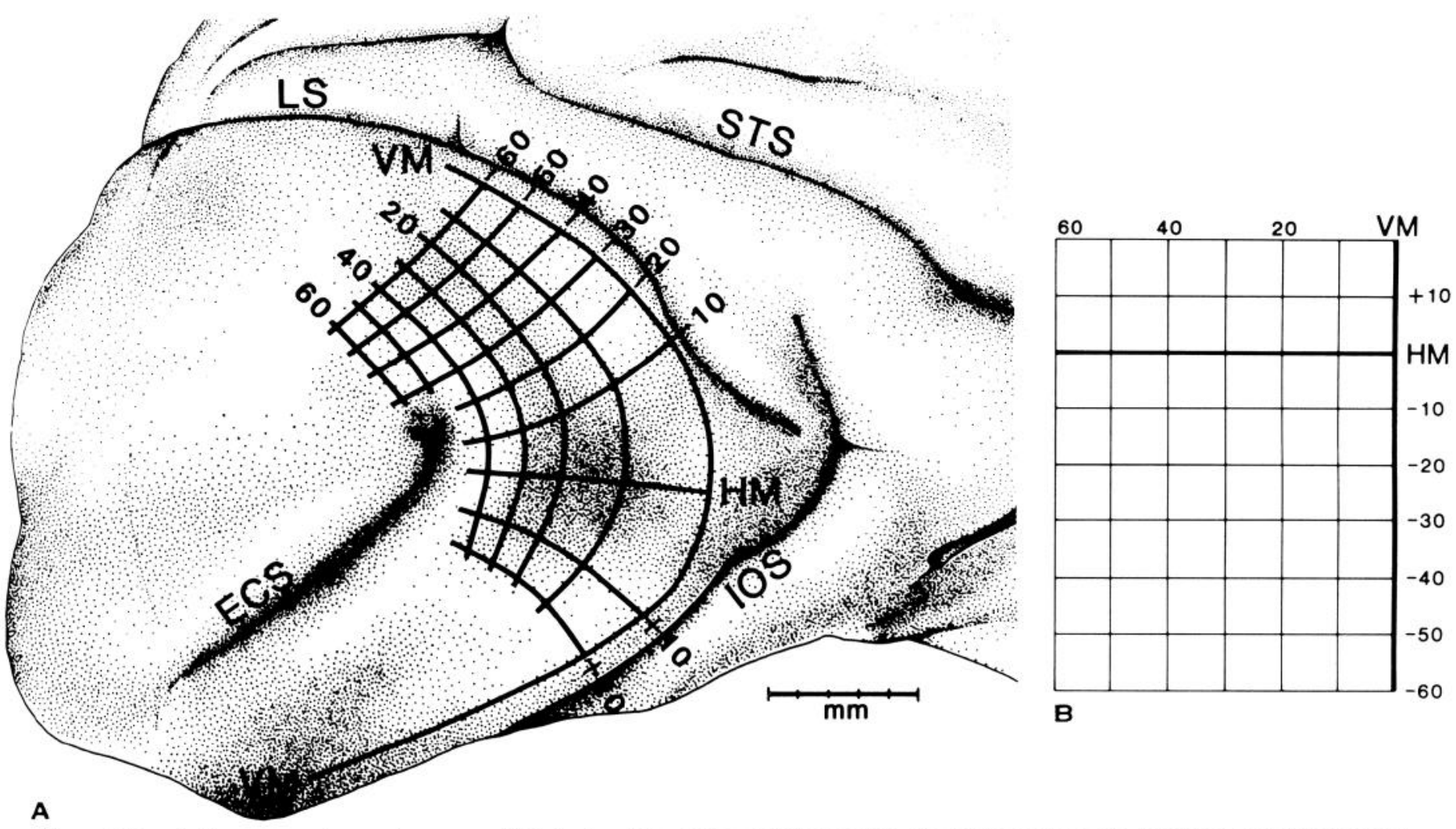

Figure 9. Visuotopic map superimposed upon an artist's tracing of the right occipital operculum of a rhesus monkey (LR). Azimuth and elevation lines are as in Figure 7. ECS, external calcarine sulcus; IOS, inferior occipital sulcus; LS, lunate sulcus; STS, superior temporal sulcus; HM, horizontal meridian; VM, vertical meridian. $B$, The region of visual space mapped onto the brain surface in $A$. VM, vertical meridian; $H M$, horizontal meridian. Azimuth and elevation lines at 10-min intervals are represented. 


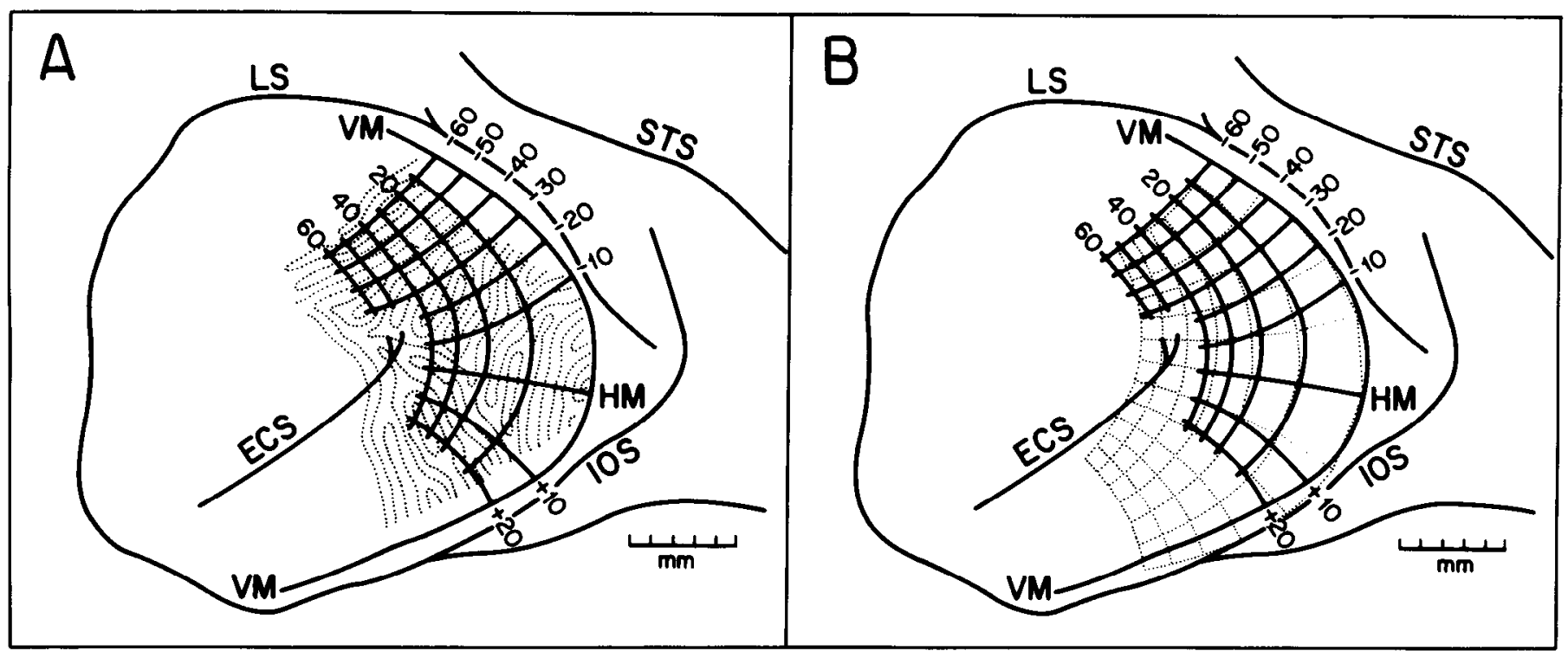

Figure 10. The visuotopic map of Figure $9 A$ compared with other striate "maps" (dotted lines). $A$, The central foveal portion of the ocular dominance stripe map of LeVay et al. (1975). $B$, The complex logarithmic mapping of Figure $9 B$, according to the equation, $w=7.7 * \ln (x+i y+0.33)$. In this equation, 7.7 is a scaling factor that adjusts the theoretical map (measured in degrees) to the actual size of the corresponding cortical tissue (measured in millimeters). Labeling is as in Figure $9 A$.

TABLE I

Analysis of pairs of penetrations made at the same cylinder location

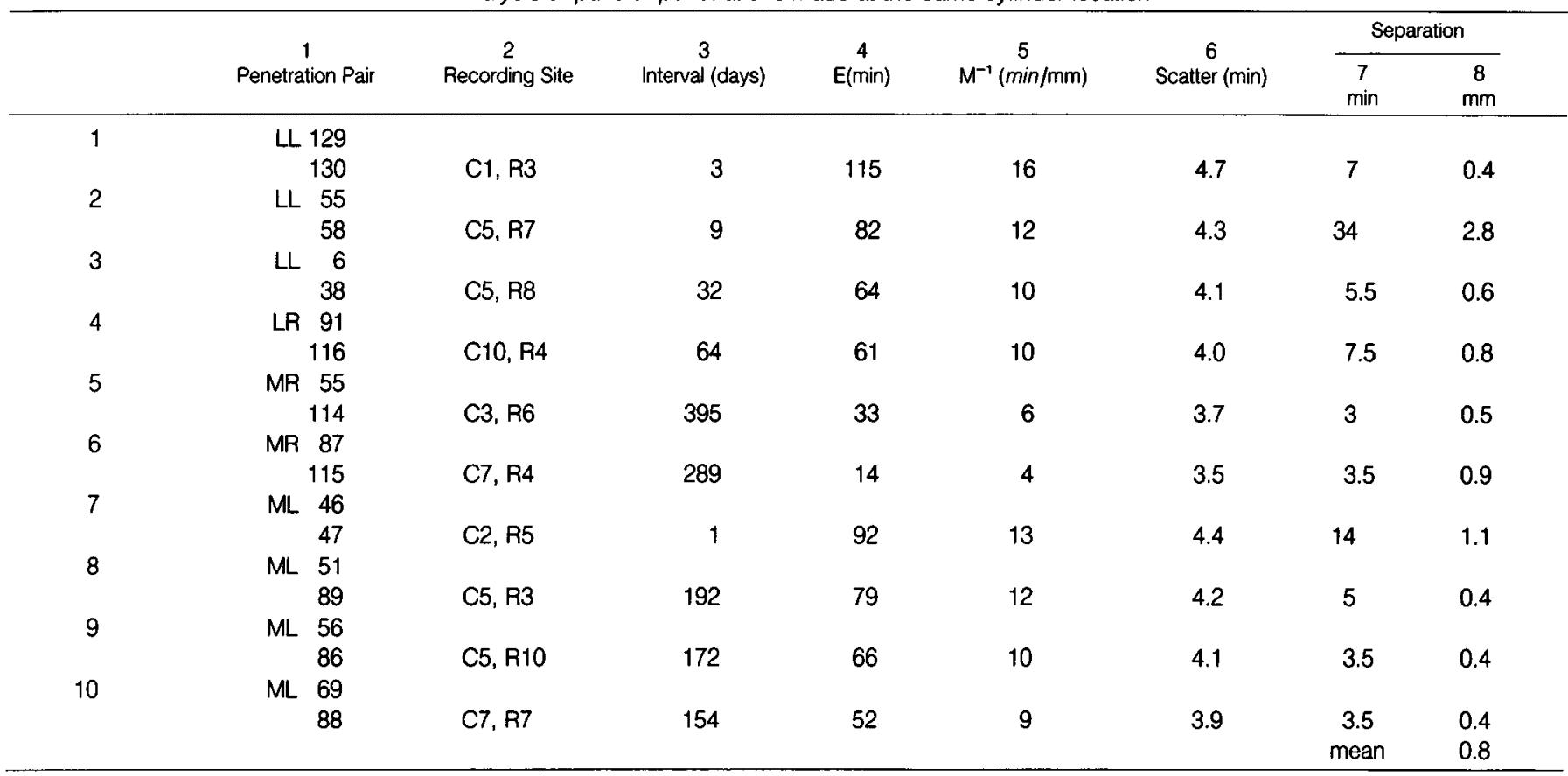

the other hand, rather substantial (about 1.5:1; see "Discussion") vertical/horizontal magnification anisotropy at eccentricities below $20 \mathrm{~min}$. At eccentricities beyond about $30 \mathrm{~min}$, vertical/horizontal magnification is more nearly isotropic.

Ocular dominance stripes. In the only available complete ocular dominance stripe map of the macaque's occipital operculum (LeVay et al., 1975) there is a tendency for the stripes to lie perpendicular to the $V 1 / \mathrm{V} 2$ border and to converge toward a line that might well be the horizontal meridian. LeVay and his colleagues (1975) proposed that the stripes ran along horizontal lines in the visual field, i.e., iso-elevation lines. They based this suggestion on the earlier published maps of Talbot and Marshall (1941) and Daniel and Whitteridge (1961). Since the present map provides considerably greater resolution in the foveal region than either of the earlier maps, we have attempted to test the proposal of LeVay et al. (1975) by superimposing our visuotopic map onto their ocular dominance stripe map.

Figure 10A shows the superimposed maps. The figure was constructed by projecting one map onto the other from an enlarger and then adjusting the enlarger until the V1/N2 borders on the two maps coincided in the central foveal region. The ocular dominance stripe map of LeVay et al. (1975) falls short of the V1/V2 border in their original figure (Fig. $8 a$ ) due to difficulty in reconstructing ocular dominance bands in regions of high curvature near the lunate and inferior occipital sulci. Only the central portion of their map is included here, since at higher eccentricities the V1/N2 borders in the two maps can no longer be made to superimpose. The viewing angles are almost certainly different in the two cases. Naturally it would be 
preferable to have a complete visuotopic map and a complete ocular dominance stripe map in the same animal at the same viewing angle. The present attempt is at least a step toward such a goal.

Examination of Figure $10 \mathrm{~A}$ does in fact suggest that the ocular dominance stripes run generally parallel to iso-elevation lines at eccentricities between 30 and $60 \mathrm{~min}$. At eccentricities of 10 and $20 \mathrm{~min}$, however, the parallel relationship begins to break down, and in the vicinity of the horizontal meridian ( $0 \mathrm{~min}$ ), at these very low eccentricities, the ocular dominance stripes run perpendicular rather than parallel to iso-elevation lines.

Figure $10 \mathrm{~A}$ therefore largely confirms the proposal of LeVay et al. (1975) and, in addition, shows that the nonparallelism of the two maps is confined to approximately the central $30 \mathrm{~min}$ in the visual field (cf. Dow and Bauer, 1984).

\section{Discussion}

A new mapping procedure. The present paper describes a method for generating a detailed visuotopic map from large numbers of closely spaced microelectrode penetrations in surface cortex of a single animal. The essential principle is to use the collection of penetration tracks as a fixed array, taking advantage of the large numbers to smooth out the effects of occasional aberrant penetrations, and then to locate this array within the tissue by means of a few marking lesions and a complete three-dimensional reconstruction of the tissue block. The procedure permits detailed comparisons of visuotopic maps obtained from different hemispheres (Fig. 7), which is important in view of differences in map details between individual animals and even between the two hemispheres of the same animal.

Evaluation and criticisms. The main advantage of the method is that it eliminates the need to obtain histological verification of every penetration. For the great majority of penetrations, one records only the $x$ and $y$ coordinates. Because individual penetrations are not marked, one is free to gather enormous amounts of information from the same tissue region. In fact, the more penetrations one makes, the better, since the number of mapping points to be averaged is thereby increased. In addition, if the problem of electrode drift with greater depth can be controlled, then one can map several tiers of buried tissue at the same time using the most completely characterized map (the V1 map in this case) as a foundation for additional maps (unpublished data). The long time periods involved allow careful studies of individual cells, thus permitting the maps to be enriched by physiological details about microarchitecture. For example, extensive studies of orientation (Bauer et al., 1983) and color (Vautin and Dow, 1985) were conducted as the data in the present report were being assembled.

The present method is quite compatible with "global" staining techniques such as 2-deoxyglucose, cytochrome oxidase, and so on (see Horton and Hubel, 1981; Tootell et al., 1982; Humphrey and Hendrickson, 1983), and provides the advantage of a large amount of physiology to be correlated with the anatomy. Furthermore, awake, behaving animals may well be more suitable than paralyzed, anesthetized animals for histochemical methods that utilize physiological activation of neuronal elements as a means of selective tissue labeling.

An obvious disadvantage is that collecting and processing the data in a single animal requires a long time. The data reported here (for two monkeys) were gathered almost continuously over a period of nearly $2 \frac{1}{2}$ years. In retrospect, it would seem advantageous in future studies to alternate short-term animals for primarily anatomical data with long-term animals for detailed physiological data.

A second disadvantage is that, without specific marks of individual recording sites, one loses some resolution with respect to specific anatomical landmarks such as tissue layers, the V1/V2 border, cytochrome oxidase puffs, ocular dominance stripes, and so on. However, sites of special interest can be specifically marked during the last 8 weeks of recording in any given animal (see Dow et al., 1981; Bauer et al., 1983).
The principal source of error with the method relates to possible discrepancies between $x, y$, and $z$ (depth) coordinates and actual histological recording sites. The issue of $z$ coordinate errors has bcen discussed previously (Bauer et al., 1983). For nine marked penetrations the microdrive reading overestimated measured histological depth by an average of $9 \%$. There are two convenient ways of estimating the size of $x, y$ errors. The most direct is to compare mark locations in the tissue (Fig. 3) with $x, y$ coordinates of marking penctrations in the corresponding cylinder map (Fig. 1). By this method, $x, y$ coordinate errors in the present study were judged to be typically less than $\pm 1 \mathrm{~mm}$. Another method, slightly less direct although more quantitative, is to compare the aggregate receptive field center locations for pairs of penetrations made at the same cylinder location. Results of the analysis of 10 such penetration pairs (open circles in Figs. $4 B, 4 D, 5 B$, and $5 D$ ) are summarized in Table I. Cylinder locations and recording intervals are shown in columns 1 to 3 . The mean receptive field center eccentricity for each penetration pair is shown in column 4, and the inverse magnification and receptive field center scatter at this eccentricity (from Dow et al., 1981, Figs. 5 and 7) are shown in columns 5 and 6. Visual field separation in minutes (obtained directly from the graphs in Figs. 4 and 5 ) is given in column 7 , and cortical separation in millimeters (computed from inverse magnification) is shown in column 8 . Here it can be seen that only two penetration pairs were separated by more than twice the receptive field scatter to be expected within a single penetration at that eccentricity, whereas four pairs were within the range of expected scatter. One clearly aberrant pair was separated by $2.8 \mathrm{~mm}$ of computed cortical distance, whereas eight were separated by less than $1 \mathrm{~mm}$. The overall mean separation was 0.8 $\mathrm{mm}$. This procedure therefore estimates $x, y$ coordinate errors to be well within $\pm 1 \mathrm{~mm}$.

In more recent experiments ( $R$. Erickson, unpublished data) the size of $x, y$ coordinate errors has been further reduced by having the microelectrode advance almost to the intact dura inside a plastic guide tube, thus assuring that the electrode enters the tissue at the proper location, and minimizing any bending as the electrode passes through the dura itself. Dural resistance can be reduced by periodic scraping (under general anesthesia), or by raising and lowering the electrode while piercing the dura.

The foveal striate visuotopic map. Schwartz (1980) has proposed, for a variety of species, that the visual field is mapped onto striate cortex according to a complex logarithmic transformation of the form, $w=\ln (x+i y+a)$. In this equation, $a$ is a constant for a given species, $x$ and $y$ are the retinal Cartesian coordinates, $i$ is the imaginary number, $\sqrt{-1}$, and $w$ is the corresponding cortical locus. Recent data extending the measurement of striate magnification into the central foveal region of the macaque (Dow et al., 1981) indicate a value of about 0.3 degree for a (Schwartz, 1983). The use of this value in the above equation results in a reasonable fit to the overall shape and known map details within the central 5 to $9^{\circ}$ (Schwartz, 1983). The development of a map of the central $1^{\circ}$ (Fig. 9A) now permits a detailed comparison of the empirical and theoretical maps for this region. Figure $10 B$ shows the theoretical map of the foveal striate region (Fig. 10B, dotted lines) predicted on the basis of a constant of 0.33 degree $(20 \mathrm{~min}$ ), which gives a slightly better fit to the empirical map (Fig. 10B, solid lines) than either 0.3 or 0.4 , based on comparison with the measured curvature of the striate outline in the foveal region. The theoretical map was scaled and aligned by eye so as to produce the best overall fit to the empirical map along both the horizontal and vertical meridia. The scaling factor used to obtain this fit (7.7) converts degrees of visual angle into millimeters of cortex.

One should keep in mind that the theoretical map is seen as it exists on a plane surface, whereas the empirical map is a plane projection from a surface that curves away from the projection plane, especially near sulci. Given this difference, the fit of the two maps in Figure $10 B$ is reasonably good, except that the central elevation 
lines determined experimentally are more eccentric than the corresponding predicted elevation lines.

Mapping the central foveal region. It should be acknowledged that the present data include rather few recording sites within the central $10 \mathrm{~min}$ of the visuotopic map (see Fig. 7). The explanation for this is that our mapping is necessarily done with the monkey fixating a target. The target itself occupies a few minutes of visual angle and interferes with mapping in that region. Projected light was used as a fixation target during early studies, but the monkey became easily distracted when visual stimuli came too close to the fixation point. An LED eliminates the distraction problem, since it remains physically attached to the screen and cannot be obscured by back-projected stimuli. The LED used in this study subtended an effective visual angle of $3.5 \mathrm{~min}$ and was mounted in an opaque annulus with an effective inside diameter of $3.5 \mathrm{~min}$ and an outside diameter of $8.5 \mathrm{~min}$. If a cell responded to the LED, its receptive field was drawn so as to include the LED. Additional portions of the receptive field were then defined on the basis of stimulus-induced responses superimposed upon a base line of LED-induced responses. The presence of the 2.5-min-wide annulus around the LED created a zone of uncertainty for mapping of central receptive fields. Because of this uncertainty we elected not to explore the central 5 min of the map.

It would of course be interesting to examine the central part of the V1 map in greater detail with the fixation point as the only stimulus. Related studies are currently in progress in our laboratory.

Magnification isotropy. Outside the foveal region Daniel and Whitteridge (1961) found no differences in magnification within six different radial segments subtending angles of $30^{\circ}$. More recently, Hubel and Wiesel and their colleagues (Hubel et al., 1974; LeVay et al., 1975; Hubel and Freeman, 1977; Hubel and Wiesel, 1977) have predicted, but have not directly demonstrated, that magnification should be 2 times as great along ocular dominance stripes as across stripes. Tootell et al. (1982), using a novel 2-deoxyglucose retinotopic mapping technique, obtained a vertical/horizontal magnification ratio of about $1.25: 1$, which they estimate should correspond to a ratio of about 1.5:1 along versus across ocular dominance stripes. It has also been noted (Dow and Bauer, 1984) that the ratio of the distances between cytochrome oxidase puffs along and across ocular dominance stripes, about 1.4:1 (Hendrickson et al., 1981; Horton and Hubel, 1981), might be an indicator of magnification anisotropy if cytochrome oxidase puffs served as retinotopic points.

Vertical/horizontal magnification isotropy can be readily measured in Figure $10 B$ by simply taking the ratio of the lengths of line segments at comparable eccentricities along the vertical and horizontal meridia. For the theoretical map in the central 20 min one obtains a vertical/horizontal anisotropy of $1.39: 1$. For the empirical map in the central 20 min the ratio is 1.50 for the inferior field and 1.43 for the superior field (mean, 1.47:1). There is thus a fairly clear vertical/horizontal magnification anisotropy of about 1.5:1 at central eccentricities in Figure 10B. However, by $60 \mathrm{~min}$ in the theoretical map the anisotropy has fallen off to about 1.2:1.

Clearly it would be of interest to obtain data from additional monkeys. Recent experiments in our laboratory (R. Erickson and B. $M$. Dow, unpublished data) have resulted in a fairly complete foveal striate map in one hemisphere of a cynomolgus monkey (Macaca fascicularis). At 0 to $20 \mathrm{~min}$ eccentricity there was a vertical/ horizontal anisotropy of 1.37:1 in the inferior field and 1.57:1 in the superior field (mean, 1.47:1). These values are in reasonably close agreement with those obtained in the present study.

The main point here is that a vertical/horizontal magnification anisotropy of about 1.5:1 is present in the central region of macaque striate cortex. Outside the foveal region vertical/horizontal magnification is more nearly isotropic.

Central foveal anisotropies. Hemispheres ML and MR of the present study, in addition to showing a vertical/horizontal magnification anisotropy at central eccentricities, also showed a clear bias for vertical orientation preference among upper layer cells at eccen- tricities below 30 min (Bauer et al., 1980; Dow and Bauer, 1984). Is there a possible relationship between these two phenomena?

A recent model (Dow and Bauer, 1984) proposes a relationship between retinotopic location in the striate map and preferred orientation at that location. In particular, the model suggests that cytochrome oxidase puffs serve as the centers of orientation hypercolumns, with iso-orientation lines arranged as spokes, rather than parallel stripes (Hubel and Wiesel, 1977). The rectangularity of the cytochrome oxidase matrix (see above) would predict that preferred orientations in interpuff zones should be shifted by about $10^{\circ}$ toward the orientation represented on the long axis of the rectangle. At eccentricities $>30 \mathrm{~min}$, the long axes of cytochrome oxidase matrix rectangles run down the centers of ocular dominance stripes and therefore follow iso-elevation lines. The model proposes that horizontal iso-orientation lines follow iso-elevation lines in this part of striate cortex. In fact, a clear bias of about $10^{\circ}$ from diagonal $\left(45^{\circ}, 135^{\circ}\right)$ orientation preference toward horizontal (i.e., $35^{\circ}, 145^{\circ}$ ) is present among top cells at eccentricities $>30 \mathrm{~min}$ (Dow and Bauer, 1984), thus providing some support for the model.

At eccentricities below $30 \mathrm{~min}$ the ocular dominance stripes do not follow iso-elevation lines, but seem rather to follow iso-eccentricity lines (Fig. 10A). If a relationship exists between retinotopic location, cytochrome oxidase puff distribution, and preferred orientation (Dow and Bauer, 1984), the altered trajectory of ocular dominance stripes in relation to retinotopic coordinate lines in the central 30 min of the striate map should be associated with a change in the organization of orientation columns. The change in anisotropy from oblique $\left(35^{\circ}, 145^{\circ}\right)$ to vertical orientation preference among top cells at these eccentricities (Bauer et al., 1980; Dow and Bauer, 1984) is at least consistent with this idea.

Do the changes in ocular dominance stripe pattern, magnification isotropy, and orientation isotropy in the central $30 \mathrm{~min}$ of striate cortex relate to any known functional specialization in the central visual field? The short wavelength-sensitive cones are essentially absent from most of this region of the retina as well (Marc and Sperling, 1977; DeMonasterio et al., 1981; Williams et al., 1981). Wald (1967) has described the "flight from blue," by means of which short wavelength light is eliminated from the central retina, thus presumably reducing chromatic aberration, and facilitaling fine pattern vision and fixation. Accommodative mechanisms are likely to play an important role in sharpening the retinal image. The reduction of chromatic aberration should be especially useful for precise accommodative fixation. Vertically oriented receptive fields are likely to be more useful for accommodative fixation than horizontally oriented receptive fields. Thus, accommodative fixation suggests itself as a possible function related to the centralmost portion of the foveal striate map.

Foveal magnification. Since the recording sites in Figure 7 form a 1-mm grid, one can readily measure local magnification at any desired map location. In hemisphere MR, for example, at 0 to 20 min eccentricity, magnification is $16.2 \mathrm{~mm} /$ degree along the horizontal meridian, $21.6 \mathrm{~mm} /$ degree along the vertical meridian in the superior field, and $24.6 \mathrm{~mm} /$ degree along the vertical meridian in the inferior field. In the cynomolgus monkey mentioned above, for which comparable data are available, corresponding values (also for 0 to $20 \mathrm{~min}$ eccentricity) are $9.0,14.1$, and $12.3 \mathrm{~mm} /$ degree. Clearly, the rather broad range of earlier estimates for foveal magnification in macaque striate cortex (summarized in Van Essen et al., 1984) represents a combination of anisotropies for various meridia (horizontal versus vertical, superior vertical versus inferior vertical) and differences between individuals and/or species. It would clearly be of interest to know whether foveal magnification in a given hemisphere is fixed in relation to total striate surface area in that hemisphere, or whether foveal magnification as a proportion of total striate area varies from individual to individual (and between hemispheres in the same individual), possibly in relation to visual experience. 


\section{References}

Allman, J. M., and J. H. Kaas (1971) A representation of the visual field in the caudal third of the middle temporal gyrus of the owl monkey (Aotus trivirgatus). Brain Res. 31: 85-105.

Allman, J. M., and J. H. Kaas (1974) A crescent-shaped cortical visual area surrounding the middle temporal area (MT) in the owl monkey (Aotus trivirgatus). Brain Res. 81: 199-213.

Bauer, R., B. M. Dow, and R. G. Vautin (1980) Laminar distribution of preferred orientations in foveal striate cortex of the monkey. Exp. Brain Res. 41: 54-60.

Bauer, R., B. M. Dow, A. Z. Snyder, and R. Vautin (1983) Orientation shift between upper and lower layers in monkey visual cortex. Exp. Brain Res. 50: $133-145$

Braitenberg, V., and C. Braitenberg (1979) Geometry of orientation columns in the visual cortex. Biol. Cybernet. 33: 179-186.

Daniel, P. M. and D. Whitteridge (1961) The representation of the visual field in the cerebral cortex in monkeys. J. Physiol. (Lond) 159: 302-321.

DeMonasterio, F. M. S. J. Schein, and E. P. McCrane (1981) Staining of blue-sensitive cones of the macaque retina by a fluorescent dye. Science 213: $1278-1281$.

Dow, B. M. and R. Bauer (1984) Retinotopy and orientation columns in the monkey: A new model. Biol. Cybernet. 49: 189-200.

Dow, B. M. R. Bauer, and R. G. Vautin (1979) Extrastriate color cells in the awake monkey. Soc. Neurosci. Abstr. 5: 782.

Dow, B. M., A. Z. Snyder, R. G. Vautin, and R. Bauer (1981) Magnification factor and receptive field size in foveal striate cortex of the monkey. Exp. Brain Res. 44: 213-228.

Evarts, E. V. (1966) Methods for recording activity of individual neurons in moving animals. Methods Med. Res. 11: 241-250.

Guld, C., and A. Bertulis (1976) Representation of fovea in the striate cortex of vervet monkey, Cercopithecus aethiops pygerythrus. Vision Res. 16: 629-631.

Hendrickson, A E., S. P. Hunt, and J. Y. Wu (1981) Immunocytochemical localization of glutamic acid decarboxylase in monkey striate cortex. Nature 292: 605-607.

Horton, J. C., and D. H. Hubel (1981) Regular patchy distribution of cytochrome oxidase staining in primary visual cortex of macaque monkey. Nature 292: 762-764.

Hubel, D. H., and D. C. Freeman (1977) Projection into the visual field of ocular dominance columns in macaque monkey. Brain Res. 122: 336343.
Hubel, D. H. and T. N. Wiesel (1972) Laminar and columnar distribution of geniculo-cortical fibers in the macaque monkey. J. Comp. Neurol. 146: 421-450.

Hubel, D. H., and T. N. Wiesel (1977) Functional architecture of macaque monkey visual cortex. Proc. R. Soc. Lond. (Biol.) 198: 1-59.

Hubel, D. H., T. N. Wiesel, and S. LeVay (1974) Visual-field representation in layer IVC of monkey striate cortex. In Society for Neuroscience Program and Abstracts 1974, p. 264, Society for Neuroscience, Bethesda, MD.

Humphrey, A. L., and A. E. Hendrickson (1983) Background and stimulusinduced patterns of high metabolic activity in the visual cortex (area 17) of the squirrel and macaque monkey. J. Neurosci. 3: 345-358.

LeVay, S., D. H. Hubel, and T. N. Wiesel (1975) The pattern of ocular dominance columns in macaque visual cortex revealed by a reduced silver stain. J. Comp. Neurol. 159: 559-576.

Marc, R. E., and H. G. Sperling (1977) Chromatic organization of primate cones. Science 196: 454-456.

Schwartz, E. L. (1980) A quantitative model of the functional architecture of human striate cortex with application to visual illusion and cortical texture analysis. Biol. Cybernet. 37: 63-76.

Schwartz, E. L. (1983) Cortical mapping and perceptual invariance: A reply to Cavanagh. Vision Res. 8: 831-835.

Talbot, S. A., and W. H. Marshall (1941) Physiological studies on neural mechanisms of visual localization and discrimination. Am. J. Ophthalmol. 24: $1255-1263$.

Tootell, R. B. H., M. S. Silverman, E. Switkes, and R. L. DeValois (1982) Deoxyglucose analysis of retinotopic organization in primate striate cortex. Science 218: $902-904$

Van Essen, D. C. (1979) Visual areas of the mammalian cerebral cortex. Annu. Rev. Neurosci. 2: 227-263.

Van Essen. D. C., W. T. Newsome, and J. H. R. Maunsell (1984) The visual field representation in striate cortex of the macaque monkey: Asymmetries, anisotropies, and individual variability. Vision Res. 24: 429-448

Vautin, R. G., and B. M. Dow (1985) Color cell groups in foveal striate cortex of the behaving macaque. J. Neurophysiol., in press.

Wald, G. (1967) Blue-blindness in the normal fovea. J. Opt. Soc. Am. 57: 1289-1301.

Weller, R. E., and J. H. Kaas (1983) Retinotopic patterns of connections of area 17 with visual areas V2 and MT in macaque monkeys. J. Comp. Neurol. 220: 253-279.

Williams, D. R., D. I. A. MacLeod, and M. M. Hayhoe (1981) Foveal tritanopia. Vision Res. 21: 1341-1356.

Zeki, S. M. (1969) Representation of central visual fields in prestriate cortex of monkey. Brain Res. 14: 271-291. 